\title{
COMPLEX-PARAMETER INTEGRAL ITERATIONS OF CARATHEODORY MAPS
}

\author{
KUNLE OLADEJI BABALOLA AND MASHOOD SIDIQ
}

\begin{abstract}
Recent studies in the class of Bazilevič maps as a whole has compelled the development, in this work, of certain complex-parameter integral iterations of Caratheodory maps. The iterations are employed in a similar manner as in [1] to study a certain subfamily of those Bazilevič maps.
\end{abstract}

\section{Background}

Recently, Babalola in [2] devised a new approach to the study, as a whole, of the well known schlicht Bazilevič maps defined as

$$
f(z)=\left\{\frac{\alpha}{1+\beta^{2}} \int_{0}^{z}[p(t)-i \beta] t^{-\left(1+\frac{i \alpha \beta}{1+\beta^{2}}\right)} g(t)^{\left(\frac{\alpha}{1+\beta^{2}}\right)} d t\right\}^{\frac{1+i \beta}{\alpha}}
$$

in the sense that the parameter $\beta$ is no longer assumed zero. In the representation (1.1), $z$ lies in the unit disk $E=\{z:|z|<1\}, f(z)$ is a regular function of the form $f(z)=z+a_{2} z^{2}+\cdots$ normalized by $f(0)=0$ and $f^{\prime}(0)=1$ whose class is designated $A$. The subclass of $A$ that contains schlicht maps only is denoted by $S$. The map $g \in S$ is starlike (that is $\operatorname{Re} z g^{\prime}(z) / g(z)>$ 0 ). The map $p(z)=1+c_{1} z+\cdots$ is Caratheodory (that is $p(0)=1$ and $\operatorname{Re} p(z)>0$ ) and its family is denoted by $P$. The parameters $\alpha$ and $\beta$ are real with $\alpha>0$ and all powers meaning principal determinations only.

The method of analysis employed by Babalola in [2] involved the modification of the normalization of the Caratheodory maps as $h(z)=p(z)+i \mu / \eta=1+i \mu / \eta+c_{1} z+\cdots$ where $\mu$ and $\eta$ are real with $\eta>0, \lambda=\eta+i \mu$ and $p \in P$; and denoted the class of $h(z)$ by $P_{\lambda}$, which for convenience we shall replace by $H_{\lambda}$ in this paper. He thus defined a new class $B(\lambda, g)$ of maps satisfying

$$
\frac{z\left(f(z)^{\lambda}\right)^{\prime}}{\eta z^{i \mu} g(z)^{\eta}} \in H_{\lambda}
$$

2010 Mathematics Subject Classification. 30 C45.

Key words and phrases. Caratheodory maps, Bazilevič maps, Integral iterations.

Corresponding author: Kunle Oladeji Babalola. 
The new definition therefore includes the Bazilevič maps as the case $\lambda=\alpha /(1+i \beta)$.

Using the Salagean derivative $D^{n}$ defined as

$$
D^{n} f(z)=D\left(D^{n-1} f(z)\right)=z\left[D^{n-1} f(z)\right]^{\prime}
$$

with $D^{0} f(z)=f(z)$ [7], Babalola in [3] further generalized (1.2) as $B_{n}(\lambda, g)$ consisting of maps $f(z)$ which satisfy

$$
\frac{D^{n} f(z)^{\lambda}}{\eta \lambda^{n-1} z^{i \mu} g(z)^{\eta}} \in H_{\lambda}
$$

and then chose $g(z)=z$ for which he obtained a number of properties of $B_{n}(\lambda) \equiv B_{n}(\lambda, z)$.

In the present paper, we shall study (1.3) also for $g(z)=z$ and $h(z)=p(z)+i \mu / \eta$ with Re $p(z)>\gamma, 0 \leq \gamma<1$ and $H_{\lambda}(\gamma)$ is designated the class of such functions. Hence for some $h \in H_{\lambda}(\gamma)$, we have

$$
\frac{D^{n} f(z)^{\lambda}}{\eta \lambda^{n-1} z^{\lambda}}=1+i \frac{\mu}{\eta}+(1-\gamma) \sum_{k=1}^{\infty} c_{k} z^{k}
$$

so that

$$
D^{n} f(z)^{\lambda}=\lambda^{n} z^{\lambda}+(1-\gamma) \sum_{k=1}^{\infty} \eta \lambda^{n-1} c_{k} z^{\lambda+k} .
$$

Applying the Salagean integral, $I_{n}$, also defined in [7] as

$$
I_{n}=I\left(I_{n-1} f(z)\right)=\int_{0}^{z}\left[\left(I_{n-1} f(t)\right) / t\right] d t
$$

with $I_{0} f(z)=f(z)$, and with some computation, we obtain

$$
\frac{\lambda f(z)^{\lambda}}{\eta z^{\lambda}}-i \frac{\mu}{\eta}=1+(1-\gamma) \sum_{k=1}^{\infty}\left(\frac{\lambda}{\lambda+k}\right)^{n} c_{k} z^{k} .
$$

The right-hand side of the above equation is

$$
p_{\lambda, n}(z)=\frac{\lambda}{z^{\lambda}} \int_{0}^{z} t^{\lambda-1} p_{\lambda, n-1}(t) d t,
$$

with $p_{\lambda, 0}(z)=p(z)$ is a Caratheodory map of order $\gamma$, and it is the justification for our study. We call $p_{\lambda, n}(z)$ the complex-parameter $n$-th integral iterations of $p \in P(\gamma)$, and its class is designated by $P_{\lambda, n}(\gamma)$. Throughout this paper, the word 'iterations' shall imply the phrase ' $n$-th complex-parameter integral iterations'. 


\section{The iterations of $P(\gamma)$}

First, we observe that the new iterations of Caratheodory maps of order $\gamma$ agrees in structure with the iterations in [1] and fortunately the complex parameter $\lambda$ has the desired positive real part. Therefore since $p_{0}(z)=p \in P(\gamma)$, then the iterations $p_{\lambda, n}(z)$ is analytic and $p_{\lambda, n}(0)=1$. It follows from (1.5) that if $p \in P(\gamma)$, then

$$
p_{\lambda, n}(z)=1+\sum_{k=1}^{\infty} c_{n, k} z^{k} \text { where } c_{n, k}=(1-\gamma)\left(\frac{\lambda}{\lambda+k}\right)^{n} c_{k}
$$

and therefore

$$
\left|c_{n, k}\right| \leq 2(1-\gamma)\left|\frac{\lambda}{\lambda+k}\right|^{n}
$$

With $p_{0}(z)=L_{0}(z)=[1+(1-2 \gamma) z] /(1-z)$, then the iterations, $L_{\lambda, n}(z)$, of the Moebius map is given as

$$
L_{\lambda, n}(z)=\frac{\lambda}{z^{\lambda}} \int_{0}^{z} t^{\lambda-1} L_{\lambda, n-1}(t) d t=1+2(1-\gamma) \sum_{k=1}^{\infty}\left(\frac{\lambda}{\lambda+k}\right)^{n} z^{k} .
$$

The iterations $L_{\lambda, n}(z)$ plays a central role with respect to the extremal problem.

As noted in [1], we remark that for complex parameters $\lambda$ and $v$ both having positive real parts, if we denote $p_{\lambda, n}(z)$ by $\chi_{n}^{(\lambda)}(p(z))$, then for any $p \in P(\gamma)$ and $m, n \in N_{0}$, we have

$$
\chi_{m}^{(v)}\left(\chi_{n}^{(\lambda)}(p(z))\right)=\chi_{n}^{(\lambda)}\left(\chi_{m}^{(v)}(p(z))\right)
$$

and for $\lambda=v$, it yields

$$
\chi_{n}^{(\lambda)}\left(\chi_{m}^{(v)}(p(z))\right)=\chi_{m}^{(v)}\left(\chi_{n}^{(\lambda)}(p(z))\right)=\chi_{n+m}^{(\lambda)}(p(z))
$$

We note here that the case $\mu=0, \eta>0$ and $\gamma=0$ yields $P_{\eta, n}(0) \equiv P_{n}$, the iterated integral transforms of Caratheodory maps defined in [1].

Next, we characterize the new iterations, $p_{\lambda, n}(z)$. The proofs follow mutatis mutandis as in [1]. However we again give the proofs for completeness and clarity of this paper, noting that $\operatorname{Re} \lambda>0$ where appropriate.

Theorem 1. Let $\gamma \neq 1$ be a non negative real number. Then for $n \in N$

$$
\operatorname{Re} p_{\lambda, n-1}(z)>\gamma \text { implies Re } p_{\lambda, n}(z)>\gamma, \text { for } 0 \leq \gamma<1
$$

and

$$
\operatorname{Re} p_{\lambda, n-1}(z)<\gamma \text { implies Re } p_{\lambda, n}(z)<\gamma, \text { for } \gamma>1
$$


Proof. On differentiation of (1.5), we have

$$
\lambda z^{\lambda-1} p_{\lambda, n}(z)+z^{\lambda} p_{\lambda, n}^{\prime}(z)=\lambda z^{\lambda-1} p_{n-1}(z)
$$

which yields

$$
p_{\lambda, n}(z)+\frac{z p_{\lambda, n}^{\prime}(z)}{\lambda}=p_{n-1}(z)
$$

It follows by the proof of Theorem 3.1 in [1] that

$$
\operatorname{Re}\left(p_{\lambda, n}(z)+\frac{z p_{\lambda, n}^{\prime}(z)}{\lambda}\right)=\operatorname{Re} p_{n-1}(z)>\gamma .
$$

This implies that $\operatorname{Re} p_{\lambda, n}(z)>\gamma$ for $0 \leq \gamma<1$ and

$$
\operatorname{Re}\left(p_{\lambda, n}(z)+\frac{z p_{\lambda, n}^{\prime}(z)}{\lambda}\right)=\operatorname{Re} p_{n-1}(z)<\gamma .
$$

This implies that $\operatorname{Re} p_{\lambda, n}(z)<\gamma$ for $\gamma>1$.

\section{Corollary 1.}

$$
P_{\lambda, n}(\gamma) \subset P(\gamma), n \in N
$$

Proof. Since $p_{0}(z)=p(z) \in P(\gamma)$, then $\operatorname{Re} p(z)>\gamma$. Hence by Theorem 1, we have, $\operatorname{Re} p_{\lambda, 1}(z)>$ $\gamma$. Also $p_{1}(z) \in P(\gamma)$, thus $\operatorname{Re} p_{\lambda, 2}(z)>\gamma$ and so on for all $n \in N$. Therefore $P_{\lambda, n}(\gamma) \subset P(\gamma)$.

\section{Theorem 2.}

$$
P_{\lambda, n+1}(\gamma) \subset P_{\lambda, n}(\gamma), n \in N
$$

Proof. Let $p_{\lambda, n+1} \in P_{\lambda, n+1}(\gamma)$, hence, there exist $p \in P(\gamma)$ such that

$$
p_{\lambda, n+1}(z)=\chi_{n+1}^{(\lambda)}(p(z))
$$

as earlier noted, then $p_{\lambda, n+1}(z)=\chi_{n}^{(\lambda)}\left(\chi_{1}^{(\lambda)}(p(z))\right)$ and using Corollary 1 , we have $\chi_{1}^{(\lambda)}(p(z)) \in$ $P(\gamma)$. Hence $p_{\lambda, n+1}$ is the $n t h$ integral transform of a function in $P(\gamma)$, that is, $p_{\lambda, n+1}$ belongs to $P_{\lambda, n}$. Therefore $P_{\lambda, n+1}(\gamma) \subset P_{\lambda, n}(\gamma)$.

Corollary 2. Let $p \in P(\gamma)$ and $\operatorname{Re} \lambda+c>0$. Then

$$
q(z)=1+(1-\gamma)(\lambda+c) \sum_{k=1}^{\infty} \frac{c_{k}}{\lambda+c+k} z^{k}, \quad z \in E
$$

is also in $P(\gamma)$. 
Proof. Let $q(z) \in P_{\lambda+c, 1}(\gamma)$, then we have

$$
q(z)=p_{\lambda+c, 1}(z)=\frac{\lambda+c}{z^{\lambda+c}} \int_{0}^{z} t^{\lambda+c-1} p_{0}(t) d t .
$$

Hence

$$
q(z)=1+(1-\gamma)(\lambda+c) \sum_{k=1}^{\infty} \frac{c_{k}}{\lambda+c+k} z^{k}
$$

is also in $P(\gamma)$.

In the next result we shall proof the surbordination $p_{\lambda, n} \prec L_{\lambda, n}$ using the technique of Briot-Bouquet differential subordination. A function $p \in P$ is said to satisfy the Briot-Bouquet differential subordination if

$$
p(z)+\frac{z p^{\prime}(z)}{\eta p(z)+\gamma} \prec h(z), \quad z \in E
$$

where $\eta$ and $\gamma$ are complex constants and $h(z)$ a complex function satisfying $h(0)=1$, and $\operatorname{Re}(\eta h(z)+\gamma)>0$ in $E$. It is well known that if $p \in P$ satisfies the Briot-Bouquet differential subordination, then $p(z)<h(z)[5]$.

A univalent function $q(z)$ is said to be a dominant of (2.3) if $p(z) \prec q(z)$ for all $p(z)$ satisfying (2.3). If $\widetilde{q}(z)$ is a dominant of (2.3) and $\widetilde{q}(z) \prec q(z)$ for all dominants $q(z)$ of (2.3), then $\widetilde{q}(z)$ is said to be the best dominant of (2.3).

Theorem 3. $p_{\lambda, n} \prec L_{\lambda, n}$.

From (2.2) we have

$$
p_{\lambda, 1}(z)+\frac{z p_{\lambda, 1}^{\prime}(z)}{\lambda}=p(z) .
$$

Now since $p \in P$, then $p<L_{0}(z)=(1+(1-2 \gamma) z) /(1-z)$ so that

$$
p_{\lambda, 1}(z)+\frac{z p_{\lambda, 1}^{\prime}(z)}{\lambda} \prec L_{0}(z) .
$$

However the differential equation

$$
q(z)+\frac{z q^{\prime}(z)}{\lambda}=L_{0}(z)
$$

has univalent solution

$$
q_{\gamma}(z)=\frac{\lambda}{z^{\lambda}} \int_{0}^{z} t^{\lambda-1} L_{0}(t) d t=L_{\lambda, 1}(z)
$$

which, by the principle of the Briot-Bouquet differential subordination, is the best dominant. Similarly, from (2.2),

$$
p_{\lambda, 2}(z)+\frac{z p_{\lambda, 2}^{\prime}(z)}{\lambda} \prec L_{\lambda, 1}(z),
$$


while the differential equation

$$
q(z)+\frac{z q^{\prime}(z)}{\lambda}=L_{\lambda, 1}(z)
$$

also has univalent solution

$$
q_{\gamma}(z)=\frac{\lambda}{z^{\lambda}} \int_{0}^{z} t^{\lambda-1} L_{\lambda, 1}(t) d t=L_{\lambda, 2}(z)
$$

which again, by the principle of the Briot-Bouquet differential subordination, is the best dominant. Continuing, we have that

$$
p_{\lambda, n}(z)+\frac{z p_{\lambda, n}^{\prime}(z)}{\lambda} \prec L_{\lambda, n-1}(z)
$$

with the differential equation

$$
q(z)+\frac{z q^{\prime}(z)}{\lambda}=L_{\lambda, n-1}(z)
$$

having univalent solution

$$
q_{\gamma}(z)=\frac{\lambda}{z^{\lambda}} \int_{0}^{z} t^{\lambda-1} L_{\lambda, n-1}(t) d t=L_{\lambda, n}(z) .
$$

This implies that $p_{\lambda, n} \prec L_{\lambda, n}$ which completes the proof.

Theorem 4. $P_{\lambda, n}(\gamma)$ is a convex set.

Proof. Let $p_{\lambda, n}(\gamma), q_{\lambda, n}(\gamma) \in P_{\lambda, n}(\gamma)$. Then for non negative real numbers $\tau_{1}$ and $\tau_{2}$ with $\tau_{1}+$ $\tau_{2}=1$, we have

$$
\tau_{1} p_{\lambda, n}(z)+\tau_{2} q_{\lambda, n}(z)=\frac{\lambda}{z^{\lambda}} \int_{0}^{z} t^{\lambda-1}\left[\tau_{1} p_{n-1}+\tau_{2} q_{n-1}\right](t) d t
$$

with $\tau_{1} p_{\lambda, 0}(z)+\tau_{2} q_{\lambda, 0}(z)=p(z) \in P(\gamma)$.

Now for $n=1$, it yields

$$
\tau_{1} p_{\lambda, 1}(z)+\tau_{2} q_{\lambda, 1}(z)=1+(1-\gamma) \sum_{k=1}^{\infty} \frac{\lambda}{\lambda+k} c_{k} z^{k} \in P_{\lambda, n}(\gamma)
$$

Suppose, it is true for $n=v-1$, thus

$$
\tau_{1} p_{\lambda, v-1}(z)+\tau_{2} q_{\lambda, \nu-1}(z)=1+(1-\gamma) \sum_{k=1}^{\infty}\left(\frac{\lambda}{\lambda+k}\right)^{\nu-1} c_{k} z^{k} \in P_{\lambda, n}(\gamma)
$$

Now for $n=v$

$$
\begin{aligned}
\tau_{1} p_{\lambda, v}(z)+\tau_{2} q_{\lambda, v}(z) & =\frac{\lambda}{z^{\lambda}} \int_{0}^{z} t^{\lambda-1}\left[1+(1-\gamma) \sum_{k=1}^{\infty}\left(\frac{\lambda}{\lambda+k}\right)^{\nu-1} c_{k} t^{k}\right] d t \\
& =1+(1-\gamma) \sum_{k=1}^{\infty}\left(\frac{\lambda}{\lambda+k}\right)^{\nu} c_{k} z^{k} \in P_{\lambda, n}(\gamma)
\end{aligned}
$$


Therefore, $P_{\lambda, n}(\gamma)$ is a convex set.

The lower bound in the next theorem is not the best possible. We provide the proof of our estimate and follow that with our expectation.

Theorem 5. Let $p_{\lambda, n} \in P_{\lambda, n}(\gamma)$. Then

$$
\left|p_{\lambda, n}(z)\right| \leq 1+2(1-\gamma) \sum_{k=1}^{\infty}\left|\frac{\lambda}{\lambda+k}\right|^{n} r^{k},|z|=r
$$

and

$$
\operatorname{Re} p_{\lambda, n}(z) \geq 1-2(1-\gamma) \sum_{k=1}^{\infty}\left|\frac{\lambda}{\lambda+k}\right|^{n} r^{k},|z|=r .
$$

Proof. For the lower bound, then from (2.1), we have

$$
\left|p_{\lambda, n}(z)-1\right| \leq 2(1-\gamma) \sum_{k=1}^{\infty}\left|\frac{\lambda}{\lambda+k}\right|^{n} r^{k} .
$$

Hence

$$
\operatorname{Re}\left(p_{\lambda, n}(z)-1\right) \geq-2(1-\gamma) \sum_{k=1}^{\infty}\left|\frac{\lambda}{\lambda+k}\right|^{n} r^{k}
$$

so that

$$
\operatorname{Re} p_{\lambda, n}(z) \geq 1-2(1-\gamma) \sum_{k=1}^{\infty}\left|\frac{\lambda}{\lambda+k}\right|^{n} r^{k}
$$

Conjecture: Let $p_{\lambda, n} \in P_{\lambda, n}(\gamma)$. Then

$$
\operatorname{Re} p_{\lambda, n}(z) \geq 1+2(1-\gamma) \sum_{k=1}^{\infty}\left|\frac{\lambda}{\lambda+k}\right|^{n}(-r)^{k},|z|=r .
$$

\section{Some applications of $P_{\lambda, n}(\gamma)$}

Here we present several different applications of the iterations $p_{\lambda, n}$ of Caratheodory maps to the study of a new subclass $B_{n}^{\lambda}(\gamma)$ of Bazilevič maps. We begin with

Definition 3.1. A regular map $f \in A$ belongs to the class $B_{n}^{\lambda}(\gamma)$ if and only if

$$
\frac{D^{n} f(z)^{\lambda}}{\eta \lambda^{n-1} z^{\lambda}} \in P_{\lambda}(\gamma)
$$

where all parameters have their usual definitions. 
Remark 1. (i) If $\gamma=0$, we recovers the class $B_{n}(\lambda)$ studied in [3].

(ii) If $\mu=0$, we then deduce the class $B_{n}^{\eta}(\gamma)$ which is equivalent to class $T_{n}^{\eta}(\gamma)$ studied in [1, 6] with change in notation as a matter of convenience. (iii) For all $f \in B_{n}^{\lambda}(\gamma)$, Equations (1.4) and (1.5) hold for some $p \in P_{\lambda}(\gamma)$. Hence we have the following relation between $P_{\lambda, n}(\gamma)$ and $B_{n}^{\lambda}(\gamma)$ which will be used in our characterization of the class $B_{n}^{\lambda}(\gamma)$ :

Lemma 1. Let $f \in A$, then the following are equivalent:

(i) $f \in B_{n}^{\lambda}(\gamma)$,

(ii) $\frac{D^{n} f(z)^{\lambda}}{\eta \lambda^{n-1} z^{\lambda}} \in P_{\lambda}(\gamma)$,

(iii) $\frac{\lambda f(z)^{\lambda}}{\eta z^{\lambda}}-i \frac{\mu}{\eta} \in P_{\lambda, n}(\gamma)$.

Proof. If $f \in A$, then by Definition 3.1 it is clear that (i) $\Leftrightarrow$ (ii). Now (ii) is true $\Leftrightarrow$ there exist $h \in P(\gamma)$ such that

$$
\frac{D^{n} f(z)^{\lambda}}{\eta \lambda^{n-1} z^{\lambda}}=h(z)=p(z)+\frac{i \mu}{\eta}=1+\frac{i \mu}{\eta}+(\gamma) \sum_{k=1}^{\infty} c_{k} z^{k}
$$

for some $p \in P(\gamma)$, so that

$$
D^{n} f(z)^{\lambda}=\lambda^{n} z^{\lambda}+(1-\gamma) \sum_{k=1}^{\infty} \eta \lambda^{n-1} c_{k} z^{\lambda+k}
$$

Applying on (3.1) the integral operator, $I_{n}$, defined by Salagean (1983) as

$$
I_{n} f(z)=I\left(I_{n-1} f(z)\right)=\int_{0}^{z} \frac{I_{n-1} f(t)}{t} d t
$$

with $I_{0} f(z)=f(z)$ and with some computation we have

$$
\frac{\lambda f(z)^{\lambda}}{\eta z^{\lambda}}=1+i \frac{\mu}{\eta}+(1-\gamma) \sum_{k=1}^{\infty}\left(\frac{\lambda}{\lambda+k}\right)^{n} c_{k} z^{k} .
$$

Therefore

$$
\frac{\lambda f(z)^{\lambda}}{\eta z^{\lambda}}-i \frac{\mu}{\eta}=1+(1-\gamma) \sum_{k=1}^{\infty}\left(\frac{\lambda}{\lambda+k}\right)^{n} c_{k} z^{k} \in P_{\lambda, n}(\gamma) .
$$

This proves the Lemma.

Now we discuss properties of the class $B_{n}^{\lambda}(\gamma)$.

\section{Theorem 6.}

$$
B_{n+1}^{\lambda}(\gamma) \subset B_{n}^{\lambda}(\gamma)
$$


Proof. Let $f \in B_{n+1}^{\lambda}(\gamma)$. Then by Lemma 1 we have

$$
\frac{\lambda f(z)^{\lambda}}{\eta z^{\lambda}}-i \frac{\mu}{\eta}=1+(1-\gamma) \sum_{k=1}^{\infty}\left(\frac{\lambda}{\lambda+k}\right)^{n+1} c_{k} z^{k}
$$

which implies

$$
\frac{\lambda f(z)^{\lambda}}{\eta z^{\lambda}}-i \frac{\mu}{\eta} \in P_{\lambda, n+1}(\gamma)
$$

Then by Theorem 2

$$
\frac{\lambda f(z)^{\lambda}}{\eta z^{\lambda}}-i \frac{\mu}{\eta} \in P_{\lambda, n}(\gamma)
$$

Hence by Lemma 1 again, $f \in B_{n}^{\lambda}(\gamma)$.

Corollary 3. For $n \geq 1, B_{n}^{\lambda}(\gamma)$ consists of schlicht maps only.

Proof. For $n \geq 1$, by Theorem 7 and Remark 3 we have $B_{n+1}^{\lambda}(\gamma) \subset B_{n}^{\lambda}(\gamma)$ and $B_{n}^{\lambda}(\gamma) \subset B_{n}(\lambda)$, which is known to consist only of schlicht maps for $n \geq 1$ (see Corollary 1 in [1]). Therefore the assertion follows.

Theorem 7. Let $f \in B_{n}^{\lambda}(\gamma)$. Then the integral

$$
F(z)^{\lambda}=\frac{\lambda+c}{z^{c}} \int_{0}^{z} t^{c-1} f(t)^{\lambda} d t
$$

is also in $B_{n}^{\lambda}(\gamma)$.

Proof. From (3.2) we have

$$
\frac{\lambda F(z)^{\lambda}}{\eta z^{\lambda}}-i \frac{\mu}{\eta}=\frac{\lambda+c}{z^{\lambda+c}} \int_{0}^{z} t^{\lambda+c-1}\left(\frac{\lambda f(t)^{\lambda}}{\eta t^{\lambda}}-i \frac{\mu}{\eta}\right) d t .
$$

Now suppose $f \in B_{n}^{\lambda}(\gamma)$ and let $v=\lambda+c$ be a complex number, then we have

$$
\frac{\lambda F(z)^{\lambda}}{\eta z^{\lambda}}-i \frac{\mu}{\eta}=\frac{u}{z^{u}} \int_{0}^{z} t^{u-1}\left(\frac{\lambda f(t)^{\lambda}}{\eta t^{\lambda}}-i \frac{\mu}{\eta}\right) d t .
$$

Hence, we have

$$
\frac{F(z)^{\lambda}}{\eta z^{\lambda}}-i \frac{\mu}{\eta}=\chi_{1}^{(\nu)}\left(\chi_{n}^{(\lambda)}(p(z))\right)=\chi_{n}^{(\lambda)}\left(\chi_{1}^{(\nu)}(p(z))\right) .
$$

With Corollary 1, $\chi_{1}^{(\nu)}(p(z)) \in P(\gamma)$, thus $\chi_{n}^{(\lambda)}\left(\chi_{1}^{(v)}(p(z))\right) \in P_{\lambda, n}(\gamma)$, which implies that $\frac{\lambda F(z)^{\lambda}}{\eta z^{\lambda}}-$ $i \frac{\mu}{\eta} \in P_{\lambda, n}(\gamma)$. Therefore by Lemma $1, F \in B_{n}^{\lambda}(\gamma)$. 
Theorem 8. Let $f \in B_{n}^{\lambda}(\gamma)$ and define

and

$$
M_{T}\left(n, \lambda^{*}, \gamma, r\right)=1+2(1-\gamma) \sum_{k=1}^{\infty} \lambda_{k}^{*} r^{k}
$$

$$
m_{T}\left(n, \lambda^{*}, \gamma, r\right)=1-2(1-\gamma) \sum_{k=1}^{\infty} \lambda_{k}^{*} r^{k}
$$

where $\lambda^{*}=\eta|\lambda|^{n-1} /|\lambda+k|^{n}$. Then

$$
m_{T}\left(n, \lambda^{*}, \gamma, r\right) \leq\left|\left(\frac{f(z)}{z}\right)^{\lambda}\right| \leq M_{T}\left(n, \lambda^{*}, \gamma, r\right) .
$$

The inequality on the right side is sharp while the one on the left is not.

Proof. Taking $p_{\lambda, n}(z)=\frac{\lambda f(z)^{\lambda}}{\eta z^{\lambda}}-i \frac{\mu}{\eta}$ in Theorem 6, we have

$$
\frac{\lambda f(z)^{\lambda}}{\eta z^{\lambda}}-i \frac{\mu}{\eta}=1+(1-\gamma) \sum_{k=1}^{\infty}\left(\frac{\lambda}{\lambda+k}\right)^{n} c_{k} z^{k} .
$$

Then

$$
\left(\frac{f(z)}{z}\right)^{\lambda}=1+(1-\gamma) \eta \lambda^{n-1} \sum_{k=1}^{\infty} \frac{c_{k}}{(\lambda+k)^{n}} z^{k} .
$$

Using triangle inequality, then we have

$$
\begin{aligned}
\left|\left(\frac{f(z)}{z}\right)^{\lambda}\right| & \leq 1+2(1-\gamma) \eta|\lambda|^{n-1} \sum_{k=1}^{\infty} \frac{r^{k}}{|\lambda+k|^{n}} \\
& \leq 1+2(1-\gamma) \sum_{k=1}^{\infty} \lambda_{k}^{*} r^{k} .
\end{aligned}
$$

For lower bound, we have

$$
\operatorname{Re}\left(\frac{f(z)^{\lambda}}{z^{\lambda}}-1\right) \geq-2(1-\gamma) \eta|\lambda|^{n-1} \sum_{k=1}^{\infty} \frac{r^{k}}{|\lambda+k|^{n}} .
$$

Hence

$$
\operatorname{Re}\left(\frac{f(z)}{z}\right)^{\lambda} \geq 1-2(1-\gamma) \sum_{k=1}^{\infty} \lambda_{k}^{*} r^{k}
$$

which concludes the proof.

Theorem 9. Let $f \in B_{n}^{\lambda}(\gamma)$ and define

$$
M_{T}^{*}\left(n, \lambda^{* *}, \gamma, r\right)=1+2(1-\gamma) \sum_{k=1}^{\infty} \lambda_{k}^{* *} r^{k}
$$

and 


$$
m_{T}^{*}\left(n, \lambda^{* *}, \gamma, r\right)=1-2(1-\gamma) \sum_{k=1}^{\infty} \lambda_{k}^{* *} r^{k}
$$

where $\lambda^{* *}=\eta|\lambda|^{n-2} /|\lambda+k|^{n-1}$. Then

$$
m_{T}^{*}\left(n, \lambda^{* *}, \gamma, r\right) \leq\left|\frac{f(z)^{\lambda-1} f^{\prime}(z)}{z^{\lambda-1}}\right| \leq M_{T}^{*}\left(n, \lambda^{* *}, \gamma, r\right) .
$$

The inequality on the right side is sharp while the one on the left side is not.

Proof. Since $f \in B_{n}^{\lambda}(\gamma)$, then by Lemma 1 there exist $p_{\lambda, n} \in P_{\lambda, n}(\gamma)$ such that

$$
\frac{\lambda f(z)^{\lambda}}{\eta z^{\lambda}}-i \frac{\mu}{\eta}=p_{\lambda, n}(z) .
$$

Then we differentiate to get

$$
\frac{\lambda f(z)^{\lambda-1} f^{\prime}(z)}{\eta z^{\lambda-1}}-i \frac{\mu}{\eta}=p_{\lambda, n}(z)+\frac{z p_{\lambda, n}^{\prime}(z)}{\lambda} .
$$

Hence from (1.5) we have

$$
p_{\lambda, n}(z)+\frac{z p_{\lambda, n}^{\prime}(z)}{\lambda}=p_{\lambda, n-1}(z)
$$

Now substitute (3.4) in (3.3), then (3.3) now becomes

$$
\frac{\lambda f(z)^{\lambda-1} f^{\prime}(z)}{\eta z^{\lambda-1}}-i \frac{\mu}{\eta}=p_{\lambda, n-1}(z) .
$$

with

$$
p_{\lambda, n-1}(z)=1+(1-\gamma) \sum_{k=1}^{\infty}\left(\frac{\lambda}{\lambda+k}\right)^{n-1} c_{k} z^{k}
$$

Hence by triangular inequality, we have

$$
\begin{aligned}
\left|\frac{f(z)^{\lambda-1} f^{\prime}(z)}{z^{\lambda-1}}\right| & \leq 1+2(1-\gamma) \eta|\lambda|^{n-2} \sum_{k=1}^{\infty} \frac{r^{k}}{|\lambda+k|^{n-1}} \\
& \leq 1+2(1-\gamma) \sum_{k=1}^{\infty} \lambda_{k}^{* *} r^{k} .
\end{aligned}
$$

This bound is sharp. For the lower bound, we have

$$
\begin{aligned}
\frac{\lambda f(z)^{\lambda-1} f^{\prime}(z)}{\eta z^{\lambda-1}}-i \frac{\mu}{\eta}-1 & =(1-\gamma) \sum_{k=1}^{\infty}\left(\frac{\lambda}{\lambda+k}\right)^{n-1} c_{k} z^{k} . \\
\operatorname{Re}\left(\frac{f(z)^{\lambda-1} f^{\prime}(z)}{z^{\lambda-1}}-\frac{i \mu}{\eta}-1\right) & \geq-2(1-\gamma) \eta|\lambda|^{n-2} \sum_{k=1}^{\infty} \frac{r^{k}}{|\lambda+k|^{n-1}} \\
& \geq-2(1-\gamma) \sum_{k=1}^{\infty} \lambda_{k}^{* *} r^{k} .
\end{aligned}
$$


Therefore

$$
\operatorname{Re}\left(\frac{f(z)^{\lambda-1} f^{\prime}(z)}{z^{\lambda-1}}\right) \geq 1-2(1-\gamma) \sum_{k=1}^{\infty} \lambda_{k}^{* *} r^{k} .
$$

The bound is not the best possible.

Theorem 10. The set of points $\frac{\lambda f(z)^{\lambda}}{\eta z^{\lambda}}-\frac{i \mu}{\eta}$ for each $f \in B_{n}^{\lambda}(\gamma), z \in E$ is convex.

Proof. Since for each $f \in B_{n}^{\lambda}(\gamma)$, the point $\frac{\lambda f(z)^{\lambda}}{\eta z^{\lambda}}-\frac{i \mu}{\eta} \in P_{\lambda, n}(\gamma)$, then by Theorem 5 , the result follows.

\section{Consequences}

We state here the analogues of Theorems 8 to 11 for class $B(\alpha, \beta) \equiv B(\alpha, \beta, z)$ of Bazilevic maps. This is achieved by setting $\lambda=\alpha /(1+i \beta)$ so that $\eta=\alpha /\left(1+\beta^{2}\right), \mu=-\alpha \beta /\left(1+\beta^{2}\right)$; and taking $n=1$ and $\gamma=0$ in the theorems, we have respectively:

Corollary 4. Let $f \in B(\alpha, \beta)$. Then the function $F(z)$ defined by

$$
F(z)^{\frac{\alpha}{1+i \beta}}=\frac{\alpha+c(1+i \beta)}{(1+i \beta) z^{c}} \int_{0}^{z} t^{c-1} f(t)^{\frac{\alpha}{1+i \beta}} d t
$$

is also in $B(\alpha, \beta)$.

Corollary 5. Let $f \in B(\alpha, \beta)$ and define

and

$$
M_{T}(\alpha, \beta, r)=1+2 \sum_{k=1}^{\infty} \frac{\alpha}{\sqrt{\left(1+\beta^{2}\right)\left[(\alpha+k)^{2}+\beta^{2} k^{2}\right]}} r^{k}
$$

$$
m_{T}(\alpha, \beta, r)=1-2 \sum_{k=1}^{\infty} \frac{\alpha}{\sqrt{\left(1+\beta^{2}\right)\left[(\alpha+k)^{2}+\beta^{2} k^{2}\right]}} r^{k} .
$$

Then $m_{T}(\alpha, \beta, r) \leq\left|\left(\frac{f(z)}{z}\right)^{\frac{\alpha}{1+i \beta}}\right| \leq M_{T}(\alpha, \beta, r)$. The inequality on the right side is sharp while the one on the left is not.

Corollary 6. Let $f \in B(\alpha, \beta)$ and define

and

$$
M_{T}^{*}(\alpha, \beta, r)=1+2 \sum_{k=1}^{\infty} \frac{r^{k}}{\sqrt{1+\beta^{2}}}
$$

$$
m_{T}^{*}(\alpha, \beta, r)=1-2 \sum_{k=1}^{\infty} \frac{r^{k}}{\sqrt{1+\beta^{2}}} .
$$

Then $m_{T}^{*}(\alpha, \beta, r) \leq\left|\left(\frac{f(z)}{z}\right)^{\frac{\alpha-1-i \beta}{1+i \beta}} f^{\prime}(z)\right| \leq M_{T}^{*}(\alpha, \beta, r)$. The right hand side inequality is sharp while the one on the left is not.

Corollary 7. The set of points $(1-i \beta)\left(\frac{f(z)}{z}\right)^{\frac{\alpha}{1+i \beta}}+i \beta$ for each $f \in B(\alpha, \beta), z \in E$ is convex. 


\section{References}

[1] K. O. Babalola and T. O. Opoola, Iterated integral transforms of Caratheodor functions and their applications to analytic and univalent functions, Tamkang J. Math. 37 (2006), 355-366.

[2] K. O. Babalola, New Insights into Bazilevic Maps. Analele Universitatii Oradea, Fasc. Matematica, Tom, xxiii, (1), (2016). Department of Mathematics, faculty of science, University of Oradea. Romania.

[3] K. O. Babalola, New generalizations of Bazilevic maps. Journal of Classical Analysis, 8, (2), $163-170$.

[4] I. E. Bazilevič, On a class of integrability by quadratures of the equation of Loewner-Kufarev, Mat. Sb., 37 (1955), 471-476 (Russian).

[5] P. Eenigenburg, S. S. Miller, P. T. Mocanu and M. O. Reade, On a Briot-Bouquet differential subordination, Rev. Roumanie Math. Pures Appl., 29 (1984), 567-573.

[6] T. O. Opoola, On a new subclass of Univalent functions, Mathematica (Cluj), 36(1994), 59 (2), $195-200$.

[7] G. S. Salagean, Subclasses of univalent functions, Lecture Notes in Math., 1013 (1983), 362-372. SpringerVerlag, Berlin, Heidelberg and New York.

Department of Mathematics, University of Ilorin, Ilorin, Nigeria.

E-mail: kobabalola@gmail.com

E-mail: mashoodsidiq@yahoo.com 